\title{
Perfil da assistência prestada por enfermeiras obstetras ao parto e nascimento em um centro de saúde materno-infantil do Recife
}

\author{
Profile of the assistance provided by obstetric nurses to childbirth and birth in a maternal- \\ child health center in Recife
}

\begin{abstract}
Perfil de la asistencia brindada por enfermeras obstétricas al parto y al nacimiento en un centro de salud materno-infantil de Recife
\end{abstract}

Géssica Kyvia Soares de Lima ${ }^{1 *}$, Viviane Maria Gomes de Araújo ${ }^{1}$, Isly Talita Santana dos Santos ${ }^{1}$, Bárbara Bruna Fernandes de Andrade ${ }^{1}$, Raine Danyele Vieira de Sousa ${ }^{1}$, Joanna Francyne Silva de Barros ${ }^{1}$, Camila Carvalho dos Santos ${ }^{1}$, Letícia de Santana Silva ${ }^{1}$, Duana Gabrielle de Lemos Costa ${ }^{1}$, Amuzza Aylla Pereira dos Santos².

\section{RESUMO}

Objetivo: Traçar o perfil da assistência prestada por enfermeiras obstetras ao parto e nascimento num Centro de Parto Normal (CPN) Intra-hospitalar. Métodos: Estudo documental, retrospectivo, transversal e quantitativo, realizado no CPN de uma instituição filantrópica, em Recife. Foram analisados no Programa SPSS 20.0 os dados de 424 parturientes e seus recém-nascidos, registrados em planilhas e livros, no período de agosto de 2018 a agosto de 2019. O estudo foi aprovado por Comitê de Ética em Pesquisa Resultados: As parturientes $(74,05 \%)$ tinham entre 25 e 35 anos e eram secundíparas ou multíparas $(53,06 \%)$. Tiveram acompanhante $(98,34 \%)$, assistência com acesso a partograma $(57,02 \%)$, uso de exercícios de respiração, banho morno e massagem, e partos verticalizados (91,74\%). Não houve laceração perineal em $21,69 \%$; nas que apresentaram, predominou as de $1^{\circ}$ grau $(52,83 \%)$. Nenhuma parturiente foi submetida à episiotomia, e mais de $80,0 \%$ não receberam intervenções obstétricas. Os recém-nascidos tiveram clampeamento tardio do cordão $(89,37 \%)$, contato pele a pele $(96,22 \%)$, respeito à hora dourada $(89,07 \%)$ e Apgar entre 7 e 10 no $1^{\circ}$ minuto $(95,51 \%)$ e $5^{\circ}$ minuto $(99,52 \%)$. Conclusão: Esse CPN apresenta como perfil uma assistência embasada em evidências científicas atuais, alcançando resultados obstétricos e neonatais favoráveis.

Palavras-chave: Centros de saúde materno-infantil, Enfermagem obstétrica, Parto humanizado.

\begin{abstract}
Objective: To outline the profile of the assistance provided by obstetric nurses for childbirth and birth in a Normal Childbirth Center (CPN). Methods: Documentary, retrospective, transversal and quantitative study, carried out at the CPN of a philanthropic institution, in Recife. Recife. The data of 424 parturients and their newborns, recorded in spreadsheets and books, from August 2018 to August 2019 were analyzed in the SPSS 20.0 Program. The study was approved by the Research Ethics Committee. Results: The parturients (74.05\%) were between 25 and 35 years old and were secondary or multiparous (53.06\%). They had a companion (98.34\%), assistance with access to a partogram (57.02\%), use of breathing exercises, warm bath and massage, and vertical births (91.74\%). There was no perineal laceration in $21.69 \%$; in those who presented, 1 st grade $(52.83 \%)$ predominated. No parturient was submitted to an episiotomy, and more than $80.0 \%$ did not receive obstetric interventions. The newborns had late cord clamping (89.37\%), skin-to-skin contact
\end{abstract}

\footnotetext{
1 Instituto de Medicina Integral Professor Fernando Figueira (IMIP), Recife - PE.

*E-mail: gessica_kyvia@hotmail.com

2 Universidade Federal de Alagoas (UFAL), Maceió - AL.
}

SUBMETIDO EM: $8 / 2020$

PUBLICADO EM: 2/2021 
(96.22\%), respect to the golden hour (89.07\%) and Apgar score between 7 and 10 in the 1 st minute (95.51\%) and 5th minute (99.52\%). Conclusion: This CPN presents as a profile assistance based on current scientific evidence, achieving favorable obstetric and neonatal results.

Keywords: Maternal-child health centers, Obstetric nursing, Humanizing delivery.

\section{RESUMEN}

Objetivo: Perfilar el perfil de la asistencia brindada por enfermeras obstétricas para el parto y parto en un Centro de Parto Normal (CPN). Métodos: Estudio documental, retrospectivo, transversal y cuantitativo, realizado en el CPN de una institución filantrópica, en Recife. Los datos de 424 parturientas y sus recién nacidos, registrados en planillas y libros, de agosto de 2018 a agosto de 2019 fueron analizados en el Programa SPSS 20.0 El estudio fue aprobado por el Comité de Ética en Investigación Resultados: Las parturientas $(74,05 \%)$ tenían entre 25 y 35 años y eran secundarios o multíparos $(53,06 \%)$. Tuvieron acompañante $(98,34 \%)$, asistencia con acceso a partograma $(57,02 \%)$, uso de ejercicios respiratorios, baño caliente y masajes y partos verticales $(91,74 \%)$. No hubo laceración perineal en el $21,69 \%$; en los que se presentaron, predominó el primer grado $(52,83 \%)$. Ninguna parturienta fue sometida a episiotomía y más del $80,0 \%$ no recibió intervenciones obstétricas. Los recién nacidos presentaron pinzamiento tardío del cordón umbilical $(89,37 \%)$, contacto piel a piel $(96,22 \%)$, respecto a la hora dorada $(89,07 \%)$ y puntuación de Apgar entre 7 y 10 en el 1er minuto $(95,51 \%$ ) y quinto minuto $(99,52 \%)$. Conclusión: Este CPN presenta un perfil de asistencia basado en la evidencia científica actual, logrando resultados obstétricos y neonatales favorables.

Palabras clave: Centros de salud materno-infantil, Enfermería obstétrica, Parto humanizado.

\section{INTRODUÇÃO}

Ao longo da história o cenário do parto e nascimento esteve permeado por mudanças. No início, a assistência ao parto era de domínio feminino e as parteiras as únicas responsáveis por essa prática, sendo um momento vivido apenas pelas mulheres em seus domicílios. Por volta do século XX, com o aumento do desenvolvimento tecnológico, esse processo passou a ser atendido nos hospitais a partir de um paradigma biomédico, sendo frequentemente compreendido como patológico, justificando o uso de intervenções mais intensas (CASTRO RCMB et al., 2018; ROJAS D et al., 2017). Com isso, foi observado o aumento do número de cesáreas e elevadas taxas de mortalidade materna e morbidade perinatal (ZANARDO GLP et al., 2017).

Ao serem relegadas a coadjuvantes, as mulheres acabaram por sentirem-se insatisfeitas com esse modelo tecnocrático, e então, a partir dos anos 70 , passou-se a questionar práticas relacionadas ao parto e ao nascimento. No final da década de 1980, surge o movimento social pela humanização do parto e nascimento, baseado nas propostas da Organização Mundial da Saúde (OMS) que, buscava estimular o parto vaginal, a amamentação logo após o parto, o alojamento conjunto, a presença de acompanhante, a atuação de enfermeiras obstétricas na atenção ao parto normal; e recomendava diminuir intervenções desnecessárias. No final do século XX, cresce também o movimento da Medicina Baseada em Evidências (ZANARDO GLP et al., 2017; TOSTES NA e SEIDL EMF, 2016).

A fim de melhorar a assistência à gravidez, ao parto e ao puerpério e, consequentemente, diminuir os óbitos por causas evitáveis, em 1999 o Ministério da Saúde (MS) cria o Centro de Parto Normal (CPN), definindo-o como a unidade de saúde que presta atendimento humanizado e de qualidade exclusivamente ao parto normal sem distócias por enfermeiras obstétricas (ROCHA FR, et al., 2017; BRASIL, 1999).

Ele atua integrado a um estabelecimento assistencial de saúde (intra-hospitalar), ou nas imediações de uma maternidade de referência (peri-hospitalar); onde são adotadas as boas práticas de atenção ao parto e nascimento criados pela OMS e, uma assistência baseada em evidências científicas. Nesta perspectiva, o CPN é um equipamento de cuidado para a redução das taxas de cesáreas e de condições que possam causar repercussões graves à saúde da mulher (TEIXEIRA CS, et al., 2018; GARCIA LV, et al., 2017). 
Apesar do Centro de Parto Normal proporcionar tantos benefícios ao momento do parto e nascimento e, de sua existência no Brasil ter sido regulamentada desde 1999, ainda há poucas unidades instaladas no país, e consequentemente, poucos estudos sobre a assistência realizada no CPN. Para se ter uma ideia, ao final de 2016 foram instalados no Brasil 15 CPN, sendo que a meta do Plano Plurianual do Governo para os anos de 2012-2015 era de implantar 65 CPN até 2015 (VICO AF, 2017).

Diante do contexto, e motivada pela reestruturação de um CPN em Recife-PE nas dependências de um hospital filantrópico no ano de 2018, surgiu o interesse em fazer investigações sobre a assistência prestada neste estabelecimento. Assim, este estudo apresenta como objetivo traçar o perfil da assistência prestada por enfermeiras obstetras ao parto e nascimento num Centro de Parto Normal Intra-hospitalar.

\section{MÉTODOS}

Trata-se de um estudo descritivo documental, retrospectivo e transversal, de abordagem quantitativa, desenvolvido em Pernambuco, entre outubro e novembro de 2019, no CPN de uma instituição filantrópica referência no atendimento de alto risco materno-infantil no âmbito do SUS, e vinculado ao Programa de Residência em Enfermagem Obstétrica.

A população do estudo foi constituída pelas parturientes admitidas nesse CPN no período de agosto de 2018 a agosto de 2019, sendo uma amostra de 424 parturientes. Foram coletados os dados, sobre as parturientes e seus recém-nascidos, registrados em planilhas e livros do setor através de formulário confeccionado pela autora. Durante a coleta não houve perda de sujeitos.

As variáveis foram distribuídas em: Perfil sociodemográfico (idade, anos de estudo, estado civil, ocupação e residência); Perfil obstétrico (paridade, idade gestacional, número de consultas e local de pré-natal, amniorrex no internamento e dilatação do colo uterino); Práticas assistenciais (profissional que internou e assistiu ao parto, abertura de partograma, presença de acompanhante, uso de ocitocina no $1^{\circ}$ e $2^{\circ}$ estágio do trabalho de parto, amniotomia e métodos não farmacológicos mais utilizados); e Condições maternas e neonatais (posição no parto, grau de laceração, sutura, inserção de DIU, clampeamento do cordão umbilical, peso, sexo, Apgar no $1^{\circ}$ e 5은 minutos, reanimação neonatal, contato pele a pele e hora dourada).

Após a coleta, os dados foram analisados no programa Statistical Package for Social Sciences (SPSS) versão 20.0, onde as variáveis foram agrupadas e, estabelecido frequência e percentual. A pesquisa atendeu às recomendações das Resoluções 466/12 e 510/16 do Conselho Nacional de Saúde, sendo aprovada pelo Comitê de Ética em Pesquisa com Seres Humanos da instituição, registrada com CAAE: 21388919.8.0000.5201.

\section{RESULTADOS}

Foram analisados os dados de 424 parturientes e seus recém-nascidos, o que correspondeu à totalidade de pacientes que pariram no CPN desse hospital no primeiro ano de funcionamento - agosto de 2018 a agosto de 2019. Durante este período o total de admissões no CPN correspondeu a 582 parturientes, porém, $27 \%$ destas foram transferidas para o setor de alto risco, logo não foram analisadas neste estudo.

É preciso considerar ainda que por motivos de reestruturação das planilhas e livros de registro do setor alguns indicadores passaram a serem acrescentados ao longo desse período. Assim, alguns dados tiveram seus percentuais calculados em cima do número de parturientes atendidas a partir desta data em que foi incluído nas planilhas, e não do valor total da amostra.

No que concerne ao perfil sociodemográfico, observa-se que a maioria das parturientes, $74,05 \%$, estava na faixa etária de 20-35 anos de idade, 65,09\% tinha entre 8 e 11 anos de estudo, 61,31\%, não exercia nenhuma atividade remunerada ou eram estudantes e, $86,08 \%$ residiam na Região Metropolitana do Recife (RMR) (Tabela 1). 
Tabela 1 - Perfil sociodemográfico das mulheres assistidas no Centro de Parto Normal no primeiro ano de funcionamento, $n=424$.

\begin{tabular}{|c|c|c|}
\hline Variável & $\mathbf{F}$ & $\%$ \\
\hline \multicolumn{3}{|l|}{ Idade da gestante em anos } \\
\hline 10- 19 & 90 & 21,22 \\
\hline 20- 35 & 314 & 74,05 \\
\hline$>35$ & 20 & 4,71 \\
\hline \multicolumn{3}{|c|}{ Escolaridade em anos de estudo } \\
\hline Nenhum & 1 & 0,23 \\
\hline $1-3$ anos & 4 & 0,94 \\
\hline 4-7 anos & 58 & 13,67 \\
\hline 8-11 anos & 276 & 65,09 \\
\hline 12 ou mais anos & 61 & 14,38 \\
\hline Sem informação & 24 & 5,66 \\
\hline \multicolumn{3}{|l|}{ Estado civil } \\
\hline Sem companheiro & 200 & 47,16 \\
\hline Com companheiro & 200 & 47,16 \\
\hline Sem informação & 24 & 5,66 \\
\hline \multicolumn{3}{|l|}{ Ocupação } \\
\hline Sem atividade remunerada & 260 & 61,31 \\
\hline Com atividade remunerada & 43 & 10,14 \\
\hline Sem informação & 121 & 28,53 \\
\hline \multicolumn{3}{|l|}{ Residência } \\
\hline RMR & 365 & 86,08 \\
\hline Interior & 34 & 8,01 \\
\hline Outro estado & 1 & 0,23 \\
\hline Sem informação & 24 & 5,66 \\
\hline
\end{tabular}

Fonte: Lima GKS, et al., 2020; dados extraídos das planilhas e livros de registro do CPN.

Quanto ao perfil obstétrico, 53,06\% das parturientes assistidas no CPN eram secundíparas ou multíparas, $99,51 \%$ apresentou idade gestacional entre $37 \mathrm{~s}$ e $41 \mathrm{~s} 6 \mathrm{~d}$, e $73,82 \%$ delas fez seis ou mais consultas de prénatal. O local de realização do pré-natal só passou a ser registrado nas planilhas a partir de outubro de 2018 , totalizando 356 parturientes. Dessas, 45,78\% fizeram pré-natal no ambulatório do hospital no qual o CPN está inserido. Referente às características no internamento, registradas a partir de março de 2019 com um total de 183 parturientes, 27,86\% chegaram ao CPN com bolsa rota, e 69,93\%, com dilatação variando entre 5 e 8 centímetros (Tabela 2). 
Tabela 2 - Perfil obstétrico das mulheres assistidas no Centro de Parto Normal no primeiro ano de funcionamento, $\mathrm{n}=424$.

\begin{tabular}{lcc}
\hline Variável & F & $\%$ \\
\hline Primípara & & \\
\hline Sim & 199 & 46,93 \\
Não & 225 & 53,06
\end{tabular}

Idade gestacional em semanas

\begin{tabular}{lcc}
\hline$<37$ & 2 & 0,47 \\
37 a 41,6 & 422 & 99,51 \\
\hline
\end{tabular}

\section{Número de consulta pré-natal}

\begin{tabular}{lcc}
\hline$<6$ & 109 & 25,70 \\
6 ou mais & 313 & 73,82 \\
Sem informação & 2 & 0,47
\end{tabular}

\section{Onde realizou o pré-natal*}

\begin{tabular}{lcc}
\hline Neste hospital & 163 & 45,78 \\
Outro local & 193 & 54,21
\end{tabular}

\section{Amniorrex no internamento **}

\begin{tabular}{lcc}
\hline $\operatorname{Sim}$ & 51 & 27,86 \\
Não & 132 & 72,13 \\
\hline
\end{tabular}

\section{Dilatação do colo no internamento **}

\begin{tabular}{lcc}
\hline$<5$ & 38 & 20,76 \\
$5-8$ & 128 & 69,94 \\
$\geq 9$ & 16 & 8,74 \\
Sem informação & 1 & 0,54
\end{tabular}

Legenda: *Indicador registrado a partir de outubro/2018 $(\mathrm{n}=356) ;{ }^{* \star}$ Indicadores registrados a partir de março/2019 ( $\mathrm{n}=183)$.

Fonte: Lima GKS, et al., 2020; dados extraídos das planilhas e livros de registro do CPN.

O internamento foi realizado em $66,41 \%$ dos casos por enfermeiras obstetras, bem como foi a profissional responsável pela maioria das assistências ao parto, 99,2\%. O acompanhante esteve presente no trabalho de parto, parto e pós-parto de $98,34 \%$ das parturientes. O uso da ocitocina no $1^{\circ}$ estágio do trabalho de parto (TP) foi observado em 7,78\% dos casos e, no $2^{\circ}$ estágio houve um percentual de 12,26\%. Já a amniotomia foi realizada em $18,63 \%$ das assistências. A abertura do partograma foi um indicador registrado a partir de outubro de 2018. Desta forma, das 356 parturientes analisadas, 57,02\% foram acompanhadas por este instrumento. Em relação aos métodos não farmacológicos de alívio da dor, os mais utilizados foram exercícios de respiração, banho morno e massagem (dados registrados a partir de setembro de 2018, com um total de 390 parturientes) (Tabela 3). 
Tabela 3 - Práticas assistenciais no trabalho de parto, parto e nascimento das mulheres assistidas no Centro de Parto Normal no primeiro ano de funcionamento, $n=424$.

\begin{tabular}{lll}
\hline Variável & $F$ & $\%$ \\
\hline Profissional que internou & & \\
\hline Enfermeiro & 259 & 66,41 \\
Médico & 131 & 37,58
\end{tabular}

Profissional que assistiu ao parto

\begin{tabular}{lcc}
\hline Enfermeiro & 421 & 99,2 \\
Médico & 3 & 0,70 \\
\hline
\end{tabular}

\section{Abertura de partograma*}

\begin{tabular}{lll}
\hline Sim & 203 & 57,02 \\
Não & 153 & 42,97 \\
\hline
\end{tabular}

Presença de acompanhante

\begin{tabular}{lcc}
\hline Sim & 417 & 98,34 \\
Não & 7 & 1,65 \\
\hline Uso de ocitocina no 1ํ estágio do TP & & \\
\hline Sim & 33 & 7,78 \\
Não & 391 & 92,21 \\
\hline
\end{tabular}

Uso de ocitocina 2 ㅇ estágio do TP

\begin{tabular}{lcc}
\hline Sim & 52 & 12,26 \\
Não & 372 & 87,73 \\
\hline
\end{tabular}

Realização de amniotomia

\begin{tabular}{lcc}
\hline Sim & 79 & 18,63 \\
Não & 345 & 81,36 \\
\hline Métodos não farmacológicos mais utilizados ** & & 69,23 \\
\hline Massagem & 270 & 74,10 \\
Banho morno & 289 & 89,74 \\
Exercícios de respiração & 350 & 50,00 \\
Bola & 195 & 50,00 \\
Agachamento & 195 &
\end{tabular}

Legenda: *Indicador registrado a partir de outubro/2018 $(\mathrm{n}=356) ;{ }^{* \star}$ Métodos não farmacológicos mais utilizados de forma associada ou individualmente. Indicador registrado a partir de setembro/2018 ( $n=390)$.

Fonte: Lima GKS, et al., 2020; dados extraídos das planilhas e livros de registro do CPN.

As parturientes adotaram posições verticalizadas em $91,74 \%$ dos partos; $78,30 \%$ apresentaram laceração perineal, sendo mais frequentes as de $1^{\circ}$ grau, e metade (50\%) necessitou de sutura. Em 19,33\% delas, foi inserido DIU de cobre no pós-parto imediato. Após o nascimento foi proporcionado contato pele a pele imediato em $96,22 \%$ das assistências e o clampeamento do cordão umbilical ocorreu tardiamente em 89,37\% dos partos, desses $27,35 \%$ só ocorreu após a dequitação. Os recém-nascidos (RN) apresentaram índice de Apgar entre 7 e 10 no $1^{\circ}$ minuto $(95,51 \%)$ e no $5^{\circ}$ minuto $(99,52 \%)$, respectivamente. Foi necessária reanimação neonatal em $5,46 \%$ dos nascimentos e, em $89,07 \%$ foi respeitada a hora dourada. Esses últimos indicadores foram registrados a partir de março de 2019, totalizando 183 parturientes (Tabela 4). 
Tabela 4 - Condições maternas e neonatais das mulheres assistidas no Centro de Parto Normal no primeiro ano de funcionamento, $n=424$.

\begin{tabular}{|c|c|c|}
\hline Variável & $\mathrm{F}$ & $\%$ \\
\hline \multicolumn{3}{|l|}{ Posição no parto } \\
\hline Vertical & 389 & 91,74 \\
\hline Horizontal & 34 & 8,01 \\
\hline Sem informação & 1 & 0,23 \\
\hline \multicolumn{3}{|c|}{ Presença de laceração de acordo com o grau } \\
\hline Sem laceração & 92 & 21,69 \\
\hline $1^{\circ}$ & 224 & 52,83 \\
\hline $2^{\circ}$ & 101 & 23,82 \\
\hline $3^{\circ}$ & 6 & 1,41 \\
\hline $4^{\circ}$ & 1 & 0,23 \\
\hline \multicolumn{3}{|c|}{ Necessidade de sutura da laceração } \\
\hline Sim & 212 & 50 \\
\hline Não & 212 & 50 \\
\hline \multicolumn{3}{|c|}{ Inserção de DIU no pós-parto imediato } \\
\hline Sim & 82 & 19,33 \\
\hline Não & 325 & 76,65 \\
\hline Sem informação & 17 & 4 \\
\hline \multicolumn{3}{|c|}{ Clampeamento do cordão } \\
\hline Imediato & 45 & 10,61 \\
\hline Tardio & 263 & 62,02 \\
\hline Após dequitação & 116 & 27,35 \\
\hline \multicolumn{3}{|l|}{ Peso ao nascer } \\
\hline$<2500 \mathrm{~g}$ & 8 & 1,88 \\
\hline 2500 a $4000 \mathrm{~g}$ & 408 & 96,22 \\
\hline$>4000 \mathrm{~g}$ & 8 & 1,88 \\
\hline \multicolumn{3}{|l|}{ Sexo } \\
\hline Feminino & 227 & $53,53 \%$ \\
\hline Masculino & 197 & $46,46 \%$ \\
\hline \multicolumn{3}{|l|}{ Apgar 1 minuto } \\
\hline$<7$ & 19 & 4,48 \\
\hline $7-10$ & 405 & 95,51 \\
\hline \multicolumn{3}{|l|}{ Apgar 5 minuto } \\
\hline$<7$ & 2 & 0,47 \\
\hline $7-10$ & 422 & 99,52 \\
\hline \multicolumn{3}{|c|}{ Realizada reanimação neonatal * } \\
\hline Sim & 10 & 5,46 \\
\hline Não & 173 & 94,53 \\
\hline \multicolumn{3}{|c|}{ Contato pele a pele imediato } \\
\hline Sim & 408 & 96,22 \\
\hline Não & 16 & 3,77 \\
\hline \multicolumn{3}{|l|}{ Hora Dourada * } \\
\hline$\overline{S i m}$ & 163 & 89,07 \\
\hline Não & 20 & 10,92 \\
\hline
\end{tabular}

Legenda: *Indicadores registrados a partir de março/2019 ( $\mathrm{n}=183)$.

Fonte: Lima GKS, et al., 2020; dados extraídos das planilhas e livros de registro do CPN. 


\section{DISCUSSÃO}

Neste estudo, apesar das parturientes serem majoritariamente adultas jovens, houve uma frequência considerável de adolescentes, assim como na pesquisa de Inagaki ADM, et al. (2019) onde $23,1 \%$ das participantes eram adolescentes e não haviam planejado as gestações. Esses dados nos revelam que ainda há falhas no sistema de saúde brasileiro, conferindo a necessidade de mais atenção à saúde sexual e reprodutiva desse público. Similar a estudos em outros Centros de Parto Normal, as parturientes se encontravam a partir da segunda gestação (TEIXEIRA CS, et al., 2018; SANTANA AT, et al., 2019). Quase a totalidade apresentou gestação a termo; apenas duas tinham idade gestacional (IG) menor que 37 semanas, o que se deduz que já chegaram ao serviço com dilatação avançada ou, pode ter havido erro no cálculo da IG, já que o CPN em questão apresenta em seus critérios de admissão gestação a partir de 37 semanas completas.

O número mínimo de 6 consultas de pré-natal recomendado pelo MS foi alcançado pela maioria das parturientes desse CPN, assim como nos estudos de Teixeira CS, et al (2018) e Freitas JMS, et al. (2019) (BRASIL, 2012). Menos da metade delas foi acompanhada no pré-natal do próprio hospital, mas apesar deste indicador só ter sido registrado a partir de outubro de 2018, foi um resultado positivo, tendo em vista que a maioria desses atendimentos é de alto risco e, que esse CPN é um serviço de porta aberta, admitindo também parturientes acompanhadas em outras unidades de saúde públicas ou privadas.

O MS recomenda ainda a vinculação da gestante com o serviço de referência, possibilitando uma visita por volta do $6^{\circ}$ mês de gestação (BRASIL, 2012). Baseado nisso é oferecido semanalmente no pré-natal dessa instituição rodas de conversa sobre TP e parto, e visitas ao CPN. Também já foram realizadas algumas ações de pintura no ventre abertas ao público, com o intuito de aproximar e orientar as gestantes sobre os critérios de admissão.

Oferecer essas visitas traz dentre vários benefícios diminuir a ansiedade e o medo das gestantes, sobretudo, da peregrinação, comum em muitas maternidades públicas do Brasil, como em Aracajú, onde apenas $27,8 \%$ das parturientes tiveram a oportunidade de conhecer a maternidade de referência, enquanto $57,3 \%$ referiram peregrinação (INAGAKI ADM, et al., 2019). No internamento, essas parturientes chegaram com bolsa amniótica íntegra, configurando baixo risco para infecções e complicações; e em trabalho de parto ativo, assim como orienta a OMS - admissão a partir de $5 \mathrm{~cm}$ de dilatação, tendo em vista que a admissão precoce se constitui num fator predisponente para intervenções desnecessárias e parto cirúrgico (WHO, 2018).

Tanto o internamento quanto a assistência ao trabalho de parto e parto se deram sumariamente por enfermeiras obstetras. Em apenas três partos houve a necessidade da interação com o médico. Este resultado demonstra a capacidade e autonomia das enfermeiras desse CPN para atuar na assistência ao parto de baixo risco, tendo a equipe médica apenas como retaguarda, para prestar atendimento caso o CPN encaminhe ou solicite, conforme são estabelecidos pela Lei do exercício da enfermagem ㄲo 7498/86 e a Portaria ํㅡ 11 de 7 de janeiro de 2015, respectivamente (BRASIL, 1986; BRASIL, 2015).

A autonomia das enfermeiras confere benefícios não só para a parturiente, que receberá uma assistência livre de intervenções desnecessárias impostas pelo modelo biomédico vigente, mas também para a formação das residentes em enfermagem obstétrica dessa instituição.

Durante o trabalho de parto as parturientes tiveram liberdade de movimentação, foram instruídas pela equipe de enfermagem quanto aos exercícios facilitadores do parto e os métodos não farmacológicos de alívio da dor, bem como sobre as vantagens de adotar posições verticalizadas; contudo, a decisão da parturiente sempre foi respeitada, prevalecendo sobre as sugestões da equipe. Também não houve restrição quanto alimentação e ingesta hídrica.

O banho morno é citado em muitos estudos como um dos métodos mais aceitos pelas pacientes em TP. A massagem lombar tem o objetivo de suprir o estado de tensão muscular e melhorar a oxigenação uterina, sendo útil entre as contrações (DUARTE MR et al., 2019; SANTANA AT, et al., 2019; GOMES ECH e DAVIM RMB, 2018). 
Estes métodos além de terem sido usados com esse objetivo no CPN, proporcionaram um momento de maior vínculo das parturientes com a equipe e com o acompanhante, inserindo-o no processo do parir; assim como foi descrito na pesquisa de Gomes ECH e Davim RMB (2018), onde se constatou que os acompanhantes foram de grande ajuda no suporte físico e mental delas.

Apesar de várias evidências científicas comprovarem o benefício do acompanhante, como: a diminuição da duração do TP, menor vulnerabilidade à violência obstétrica (SANTANA AT, et al., 2019; BRASIL, 2012), muitos serviços de saúde ainda impõem burocracias. Um estudo realizado numa maternidade pública tradicional revelou que não foi permitido acompanhante por falta de ambiência adequada, de roupa privativa ou por ser do sexo masculino (INAGAKI ADM, et al., 2019).

No CPN em questão não são encontrados obstáculos para o cumprimento deste direito; pelo contrário, ele é estimulado desde as consultas de pré-natal, sendo permitidos dois acompanhantes até o nascimento, sem contar com a doula. Quase todas as parturientes contaram com o suporte de acompanhantes de livre escolha, estando em concordância com a Lei n¹1.108 (BRASIL, 2005).

Dentre outras práticas úteis e que devem ser estimuladas está o uso do partograma, uma ferramenta gráfica da evolução do TP (SANTANA AT, et al., 2019). No CPN desta pesquisa o partograma foi aberto pelas enfermeiras obstetras a partir de $6 \mathrm{~cm}$ de dilatação e não foram utilizadas as linhas de alerta e de ação.

Esta conduta foi baseada nos estudos internacionais mais recentes, que defendem que a fase ativa da maioria das mulheres em trabalho de parto inicia-se com dilatação cervical de $6 \mathrm{~cm}$, pois elas não dilatam $1 \mathrm{~cm} /$ hora até alcançarem $5-6 \mathrm{~cm}$; e que o uso da linha de alerta é pobre para deteç̧ão de desfechos adversos e, portanto, não deve ser utilizada (ZHANG, et al., 2010; SOUZA JP, et al., 2018).

A adesão ao partograma, entretanto, foi baixa em relação a outros CPN's que mostrou taxa superior a 90\% (SANTANA AT, et al., 2019; FREITAS JMS, et al., 2019). Contudo, é importante esclarecer que não houve registro desse dado por dois meses e que em muitas das assistências o partograma não foi aberto porque as parturientes já chegaram em trabalho de parto avançado, sem tempo hábil para realizar toque vaginal. Assim, com a exposição deste resultado, passou-se a abrir o partograma no serviço com o exame realizado na triagem, a fim de cumprir as recomendações e alcançar valores proporcionais a outros CPN's.

Ao contrário da maioria das assistências ao parto e nascimento no Brasil que são extremamente intervencionistas, os dados referentes às intervenções obstétricas nesse CPN não apresentaram taxas elevadas (SANTANA AT, et al., 2019).

A ocitocina e a amniotomia foram utilizadas de forma ponderada, avaliando sua necessidade e o momento correto de usar. Comparando com estudos em outros CPN's, onde o uso da ocitocina no TP foi cerca de 19\% e a realização de amniotomia foi menos de $6 \%$, observa-se que o CPN desta pesquisa usou menos o recurso da ocitocina e realizou mais amniotomia do que esses outros centros (SANTANA AT, et al., 2019; FREITAS JMS, et al., 2019).

Autores concordam que o enfermeiro obstetra, por apresentar uma formação menos intervencionista que os médicos, realizam menos intervenções durante o processo de parir, respeitando o fisiológico, proporcionando à mulher maior autonomia (SANTANA AT, et al., 2019). Esta afirmação está intimamente relacionada a não realização de episiotomia. Numa pesquisa realizada numa Maternidade Escola de Fortaleza-CE, foi encontrado um percentual de episiotomias de $4,8 \%$, onde os pesquisadores afirmaram que a maior parte delas foi proveniente de decisão médica, intervindo na conduta da enfermeira obstétrica (CASTRO RCMB et al., 2018).

Já em outro estudo, uma taxa de episiotomia de $16 \%$ foi realizada por enfermeiras obstetras num CPN de São Paulo (FREITAS JMS, et al., 2019). A prática da episiotomia, portanto, não pode ser exclusivamente relacionada a uma categoria profissional específica e sim ao julgamento clínico destes profissionais frente às orientações sobre seu uso seletivo.

As Organizações de Saúde não aboliram esta intervenção, mas recomendam que não ultrapasse uma porcentagem de 10\% (SANTANA AT, et al., 2019). Entretanto, deve-se analisar se de fato existe alguma 
indicação para este procedimento. Um ensaio clínico randomizado verificou que não houve diferença nos resultados maternos e neonatais entre as pacientes com episiotomia seletiva e sem episiotomia e, que a não realização dela parece ser seguro para mãe e bebê (AMORIM MM, et al., 2017). Foi sob esta perspectiva que no CPN não houve incidência de episiotomia. Sobre as lacerações perineais espontâneas, o presente estudo encontrou melhores resultados do que a pesquisa realizada em uma casa de parto em Brasília, onde ocorreram $86 \%$ de lacerações, e somente 14\% tiveram períneo íntegro (SCHETTINI NJC, et al., 2017). Contudo, o CPN apresentou valores mais elevadas do que em outros dois estudos, onde a taxa de laceração foi $70,8 \%$ e $52,37 \%$, respectivamente (CASTRO RCMB, et al., 2018; TEIXEIRA CS, et al., 2018).

É importante frisar que nenhum estudo evidenciou a realização de sutura das lacerações, o que nos leva a entender que em todas elas esse procedimento foi realizado. Nas lacerações encontradas no CPN desta pesquisa metade necessitou ser suturada por sangramento ativo, desproporção anatômica ou desejo da parturiente, enquanto a outra metade foi estabilizada só com compressão digital e/ou crioterapia, afinal, há evidências que demonstram os riscos da perineorrafia. Uma revisão sistemática que objetivou estudar a sutura em laceração de primeiro e segundo graus mostrou que aquelas realizadas em lacerações de primeiro grau podem estar mais associadas a problemas perineais do que as que não foram suturadas (ELHARMEEL SM, et al., 2015). Desta forma, é preciso haver maior senso crítico pelas equipes de saúde antes de realizála, assim como a equipe do CPN.

Entendendo o momento e o lugar convenientes para iniciar o uso de um método contraceptivo eficaz, o CPN oferece no pós-parto imediato a inserção do DIU de cobre. Embora o dispositivo esteja disponível, algumas barreiras dificultam a efetivação desta ação, como falta de conhecimento das mulheres sobre o método, o que a faz recusar a oportunidade; e o fato de apenas o médico estar autorizado a inserir o DIU e nem sempre estar disponível em tempo hábil. A falta de registro nos livros e planilhas do setor, e de estudos que evidenciem a taxa de inserção de DIU no pós-parto em outros CPN's se configura em mais barreiras para análise fidedigna deste indicador.

Sobre a assistência neonatal, o CPN aqui estudado inserido em um Hospital Amigo da Criança, contém uma equipe que une esforços para promover um atendimento humanizado, que estimula o vínculo entre família e recém-nascido e apoia o aleitamento materno. Realiza o clampeamento tardio do cordão para evitar a deficiência de ferro na primeira infância, com secção feita pelo pai/acompanhante; e posterga os cuidados de rotina do $\mathrm{RN}$ para depois de 1 hora ininterrupta de vida, período em que estará em contato pele a pele com a mãe para ajudar a regular a temperatura, respiração e choro, ajudar na amamentação e no delivramento placentário, assim como orienta o MS (CASTRO RCMB et al., 2018; TEIXEIRA CS, et al., 2018; SANTANA AT, et al., 2019). As taxas referentes ao contato pele a pele, hora dourada e clampeamento tardio do cordão foram, portanto, superiores a outros estudos (CASTRO RCMB et al., 2018; TEIXEIRA CS, et al., 2018). O índice de Apgar permaneceu igual ou superior a $7 \mathrm{com}$ taxas semelhantes a estudos realizados no Brasil e Chile (CASTRO RCMB et al., ROJAS D et al., 2017), demonstrando que as condutas obstétricas utilizadas durante todo o trabalho de parto e parto contribuíram para alcançar resultados perinatais favoráveis.

\section{CONCLUSÃO}

O estudo revelou que esse CPN apresenta como perfil uma assistência prestada majoritariamente a parturientes adultas jovens, residentes na Região Metropolitana do Recife, com acesso a acompanhamento pré-natal com número mínimo ideal de consultas, e com possibilidades de estabelecer vínculo com a maternidade de referência. O CPN em questão é composto por uma equipe de enfermeiras obstetras que atuam embasadas em evidências científicas atuais sobre humanização do parto e nascimento, com uso ponderado de intervenções, respeitando o fisiológico e o empoderamento feminino, e que busca, além do mais, melhorar suas condutas, alcançando resultados maternos e neonatais favoráveis.

\section{REFERÊNCIAS}

1. AMORIM MM, et al. Selective episiotomy vs. implementation of a non-episiotomy protocol: a randomized clinical trial. Reproductive Health, 2017; 14:55. 
2. BRASIL. Lei ํㅜ 7.498, de 25 de junho de 1986. Dispõe sobre a regulamentação do exercício da Enfermagem e dá outras providências. Brasília-DF, 1986.

3. BRASIL. Lei $n$ 11.108, de 7 de abril de 2005. Altera a Lei no 8.080, de 19 de setembro de 1990, para garantir às parturientes o direito à presença de acompanhante durante o trabalho de parto, parto e pós-parto imediato, no âmbito do Sistema Único de Saúde - SUS. Diário Oficial da União. Brasilia, 2005.

4. BRASIL. Ministério da Saúde. Atenção ao pré-natal de baixo risco. Brasília: Ministério da Saúde, $2012 ; 315$ p.

5. BRASIL. Ministério da Saúde. Portaria GM no 985, de 5 de agosto de 1999. Dispõe sobre criação, normas, critérios, atribuições, definições das características físicas e recursos humanos para o funcionamento do Centro de Parto Normal (CPN) no âmbito do SUS. Diário Oficial da União, Brasília, 1999; p 51.

6. BRASIL. Portaria ํㅜ 11, de 07 de Janeiro de 2015. Redefine as diretrizes para implantação e habilitação de Centro de Parto Normal (CPN), no âmbito do Sistema Único de Saúde (SUS), para o atendimento à mulher e ao recémnascido no momento do parto e do nascimento, em conformidade com o Componente parto e nascimento da Rede Cegonha, e dispõe sobre os respectivos incentivos financeiros de investimento, custeio e custeio mensal. Brasília, 2015.

7. CASTRO RCMB, et al. Resultados obstétricos e neonatais de partos assistidos. Rev enferm UFPE, 2018; 12(4): 832839.

8. DUARTE MR, et al. Tecnologias do cuidado na enfermagem obstétrica: contribuição para o parto e nascimento. Cogitare enferm, 2019; 24: e54164.

9. ELHARMEEL SM, et al. Surgical repair of spontaneous perineal tears that occur during childbirth versus no intervention. Cochrane Database Syst. Rev. Library, 2015; 14(8).

10. FREITAS JMS, et al. Práticas Obstétricas em Centro de Parto Normal. Escola Anna Nery, 2019; 23(4): 1-7.

11. GARCIA LV, et al. O centro de parto normal e sua contribuição para atenção obstétrica e neonatal no Brasil. Rev Elet Acervo Saúde, 2017; 7(Suppl.): S356-S363.

12. GOMES ECH, DAVIM RMB. Prática do enfermeiro obstetra quanto ao alívio da dor de parturientes. Rev Enferm UFPE, 2018; 12(12): 3426-3435.

13. INAGAKI ADM, et al. Retrato das práticas obstétricas em uma maternidade pública. Cogitare enferm, 2019; 24: e56121.

14. ROCHA FR, et al. Análise da assistência ao binômio mãe-bebê em centro de parto normal. Cogitare Enferm, 2017; (22)2: e49228.

15. ROJAS D, et al. Perfil sociodemográfico, obstétrico y neonatal de usuárias com intención de parto natural em un centro de parto integral en Chile. Matronas Prof, 2017; 18(2): e24-e31.

16. SANTANA AT, et al. Atuação de enfermeiras residentes em obstetrícia na assistência ao parto. Rev. Bras. Saúde Mater. Infant, 2019; 19(1): 145-55.

17. SCHETTINI NJC, et al. Partos normais assistidos por enfermeiras obstétricas: posição materna e a relação com lacerações perineais espontâneas. Rev enferm UFPE, 2017; 11(Supl. 2): S932-S940.

18. SOUZA JP, et al. Cervical dilatation over time is a poor predictor of severe adverse birth outcomes: a diagnostic accuracy study. BJOG, 2018; 125:991-1000.

19. TEIXEIRA CS, et al. Resultados maternos e neonatais em centro de parto normal intra-hospitalar. Enferm. Foco, 2018; 9(1): 71-75.

20. TOSTES NA, SEIDL EMF. Expectativas de Gestantes sobre o Parto e suas Percepções acerca da Preparação para o Parto. Temas Psicol, 2016; 24(2): 681-693.

21. . VICO AF. Avaliação da implantação dos Centros de Parto Normal no âmbito do Sistema Único de Saúde. Dissertação (Mestrado em Ciências) - Instituto Nacional de Saúde da Mulher, da Criança e do Adolescente Fernandes Figueira. Fundação Oswaldo Cruz, Rio de Janeiro, 2017; 79 p.

22. WHO recommendations: intrapartum care for a positive childbirth experience. Geneva: World Health Organization; 2018. Licence: CC BY-NC-SA 3.0 IGO.

23. ZANARDO GLP, et al. Violência obstétrica no Brasil: uma revisão narrativa. Psicologia \& Sociedade, 2017; 29: e155043.

24. ZHANG J, et al. Contemporary Patterns of Spontaneous Labor With Normal Neonatal Outcomes. Obstetrics \& Gynecology, 2010; 116(6). 